\title{
Quantum duality: A source point of view
}

\author{
X.-F. Qian $\oplus^{1, *}$ and G. S. Agarwal ${ }^{2, \dagger}$ \\ ${ }^{1}$ Department of Physics and Center for Quantum Science and Engineering, Stevens Institute of Technology, Hoboken, New Jersey 07030, USA \\ ${ }^{2}$ Institute for Quantum Science and Engineering, Department of Biological and Agricultural Engineering, \\ and Department of Physics and Astronomy, Texas A\&M University, College Station, Texas 77843, USA
}

(Received 22 January 2019; accepted 24 December 2019; published 4 February 2020)

\begin{abstract}
Usual quantitative studies of quantum wave-particle duality employ Young's double-slit context and treat the slits approximately as ideal point sources, i.e., neglecting practical nonperfections such as slit finite width, roughness, etc. Here, we investigate the duality of a photon that is generated from genuine point sources, i.e., a pair of nonlocally entangled two-level atoms. This justifies a free-space exact spherical wave treatment. More importantly, it allows for a systematic quantitative analysis of the source effects on a quantum particle's duality property. Surprisingly, duality is found to be a conditional phenomenon depending on the photon's atomic source. It can be tuned maximum, medium, and even minimum (completely absent) by the atomic state purity through an exact Pythagorean duality source relation. Our analysis shows another way of investigating quantum duality by accounting how the single quantum object is created. The results can be tested in various practical physical systems.
\end{abstract}

DOI: 10.1103/PhysRevResearch.2.012031

Introduction. Wave and particle are two coexisting fundamental aspects of phenomena of every single quantum object [1]. According to Bohr [2], the two aspects are contradictory but must be regarded as complementary in the sense that only the totality of them fully characterizes the possible information about the object. On the other hand, a quantum object is obviously also fully characterized by the totality of its various specific physical properties, e.g., position, momentum, spin, coherence, etc. In history, coherence (the ability to interfere) is usually treated to represent the wave property, and location (or localized position) is used to characterize the particle property. Such correspondences were first established quantitatively by Wootters and Zurek [3] and then followed by many others [4-10] to achieve a duality inequality, $V^{2}+D^{2} \leqslant 1$, between single quantum object wave interference visibility $V$ and particle location distinguishability $D$. Extended studies have explored more specific physical properties, in addition to coherence and localized position, by establishing quantitative connections of the duality inequality with photon polarization [11-16], single-particle quantum states [17], and alternative coherence measures [18,19]. Recently, it has been demonstrated that the wave-particle property is connected to the self-entanglement between path and all remaining intrinsic properties (degrees of freedom) of the same single quantum object, leading to a threeway complementary identity $[20,21], V^{2}+D^{2}+C^{2}=1$,

\footnotetext{
*xiaofeng.qian@stevens.edu

† girish.agarwal@tamu.edu
}

Published by the American Physical Society under the terms of the Creative Commons Attribution 4.0 International license. Further distribution of this work must maintain attribution to the author(s) and the published article's title, journal citation, and DOI. with $C$ being the usual entanglement measure concurrence [22].

With such a three-way identity relation, the totality of wave-particle information is now compatible with the totality of specific physical properties. Interestingly, this connection opens another perspective of the duality issue. Specific physical properties (coherence, localized position, polarization, etc.) are often determined by the source and the mechanism through which the quantum object is created. Then a natural question arises, whether wave-particle duality of a quantum object is also controlled by its source. If yes, how? Can duality be tuned? In this Rapid Communication, we provide an attempt to answer these questions by analyzing in detail duality properties in the context of the quantum object's source.

Almost all these previous quantitative studies [11-21] employ the scenario of single-particle double-slit interference, where in the analysis the two slits are often assumed to be ideal point sources of a single quantum particle. Unfortunately, no source properties such as the slit width, roughness, etc., have been taken into account in duality analyses. Accounting for these properties will lead to more accurate yet sophisticated expressions of visibility and probability distribution (see, for example, a recent detailed analysis in Ref. [23]) that will no longer generate simple exact duality inequality or identity relations.

To explore the source effects and to avoid the nonperfection of realistic double slits, here we consider a genuine two-point source, i.e., a pair of nonlocally entangled identical two-level atoms, emitting a single photon for interference. The locations of the two distant atoms serve as two possible paths (see Fig. 1 for an illustration). In this case the photon propagates in free space as a perfect spherical wave, in contrast to the diffraction waves in the double-slit case, and the roughness of the slits (causing incoherence) is accounted for by the partial coherence superposition of the atoms. Also, it should be noted 


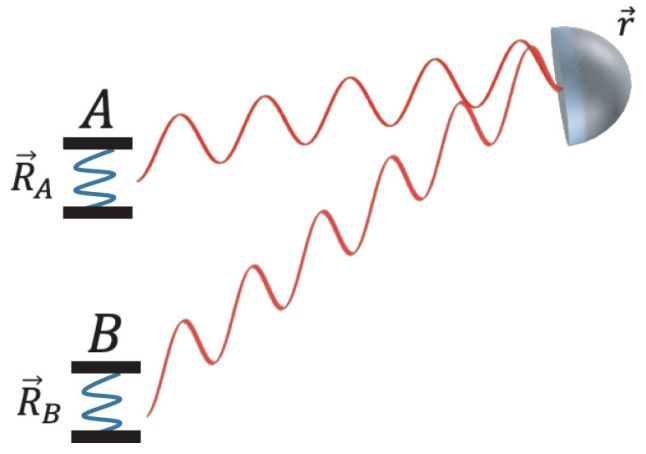

FIG. 1. Two-path interference. Two entangled atoms $A$ and $B$ (at $\vec{R}_{A}$ and $\vec{R}_{B}$, respectively) emit a photon for interference detection at $\vec{r}$.

that unlike the situation discussed in Ref. [9], there are no interactions of the single photon with external devices in its path from the source to the detector. Such a scenario was first experimentally realized by Eichmann et al. [24] with a laser beam exciting either one of the two trapped ${ }^{198} \mathrm{Hg}^{+}$ions to produce fluorescence single-photon interference (see also a detailed theoretical analyses in Refs. [25,26]). Inversely, as proposed by Cabrillo et al. [27], single-photon detection can, on the other hand, create heralded atom-atom entanglement, which was recently realized by Blatt and colleagues [28,29], with trapped ${ }^{138} \mathrm{Ba}^{+}$ions. In any of these cases, no quantitative source effect was analyzed on the duality inequality.

Generated by such a two-atom source, the single-photon which-way (particle) property directly corresponds to the information of which atom experiencing the emission, and the single-photon interference (wave) property is related to the superposed atomic state. This allows us to perform a quantification of the photon's wave and particle features through parameters of the atomic source state and retrieve the conventional duality inequality. More importantly, a detailed analysis of the source shows that the amount of information available for exchange between the photon's waveness and particleness is fully determined by the atomic state purity through an exact Pythagorean relation. An experimental configuration established in Refs. [28,29] provides a practically suitable platform for a test of our theoretical result.

Single-photon source. We consider a photon generated by the fluorescence of a pair of identical two-level atoms $(A, B)$ in an entangled single excitation state, i.e.,

$$
\left|\psi_{A B}\right\rangle=c_{a}\left|e_{A}\right\rangle\left|g_{B}\right\rangle+c_{b}\left|g_{A}\right\rangle\left|e_{B}\right\rangle,
$$

where $|e\rangle,|g\rangle$ are the excited and ground states of atom $A$ or $B$. One way of creating such an entangled state is to use Coulomb repulsion between the ions with some laser couplings [30]. Another way is to use laser pulses to excite one of two atoms from the ground state to the excited state, and then perform a single-photon detection to generate a heralded entangled atomic state, which was indirectly [24] and directly [28,29] realized in experiments. Since our analysis is based on singlephoton interference and detection, the latter procedure is more practically suitable for an experimental realization.

In practice, it is often impossible to prepare a pure atomic state (1). This may be caused by atomic thermal fluctuations, recoil due to absorption or emission of a photon by the atom, interaction with external systems and fields, etc. Therefore the two electronic energy states $\left|e_{A}\right\rangle\left|g_{B}\right\rangle$ and $\left|g_{A}\right\rangle\left|e_{B}\right\rangle$ in general should correspond to different states of the remaining degrees of freedom of the atoms as well as to the states of the external parties, i.e.,

$$
\left|\psi_{A B}\right\rangle=c_{a}\left|e_{A}\right\rangle\left|g_{B}\right\rangle|m\rangle+c_{b}\left|g_{A}\right\rangle\left|e_{B}\right\rangle|n\rangle .
$$

Here, $|m\rangle=\sum c_{m_{A}, m_{B}, m_{E}}\left|m_{A}\right\rangle\left|m_{B}\right\rangle\left|m_{E}\right\rangle$ and $|n\rangle=\sum d_{n_{A}, n_{B}, n_{E}}$ $\left|n_{A}\right\rangle\left|n_{B}\right\rangle\left|n_{E}\right\rangle$ represent two sets of states for all remaining degrees of freedom of atoms $A$ and $B$, as well as external parties $E$, indicated respectively by the subscripts.

By tracing out the states $|m\rangle$ and $|n\rangle$, i.e., all other degrees of freedom and systems, the atomic electronic energy state can be written as

$$
\rho_{A B}=\left(\begin{array}{cc}
p_{a} & \gamma \\
\gamma^{*} & p_{b}
\end{array}\right)
$$

in the basis set $\left|e_{A}\right\rangle\left|g_{B}\right\rangle,\left|g_{A}\right\rangle\left|e_{B}\right\rangle$. It is noted that state (3) derived from (2) is in fact the most general form of a mixed state. Here, $p_{a}=\left|c_{a}\right|^{2}$ is the probability of atom $A$ in an excited state and atom $B$ in the ground state, i.e., $\left|e_{A}\right\rangle\left|g_{B}\right\rangle$ (similar for $p_{b}$ ), and $\gamma=|\gamma| e^{i \varphi}=\langle m \mid n\rangle$ is the overlap of the two collective states $|m\rangle,|n\rangle$ which is restricted by the Cauchy-Schwarz inequality $|\gamma| \leqslant\left|c_{a} c_{b}\right|$.

Due to spontaneous emission, a photon will be generated by either one of the atoms $A, B$. To characterize the photon properties in terms of the source parameters, one can adopt the approach taken in Refs. [31,32]. The total single-photon field (positive frequency part) is the sum of two fields and is given as

$$
E_{A B}^{(+)}=e^{-i\left(\hat{k} \cdot \vec{R}_{A}+\phi_{A}\right)} s_{A}+e^{-i\left(k \hat{r} \cdot \vec{R}_{B}+\phi_{B}\right)} s_{B},
$$

where $k=2 \pi / \lambda=\omega / c$ denotes the wave number of the photon generated by the two atoms and $\hat{r}=\vec{r} /|\vec{r}|$ is the unit vector in the direction of the detector. The initial phases of the atomic sources $A, B$ are denoted by $\phi_{A}$ and $\phi_{B}$, respectively, and $s_{A}=\left|g_{A}\right\rangle\left\langle e_{A}\right|$ is the lowering operator of the two-level atom $A$ (similar for $s_{B}$ ).

The detection of a photon, collecting all the data when registering only one photon at a time at the detector $D$, is described with probability $p_{D}$ that is characterized by various parameters of the atomic source, i.e.,

$$
\begin{aligned}
p_{D} & \propto \operatorname{Tr}\left[\left(s_{A}^{+}+e^{-i \theta} s_{B}^{+}\right)\left(s_{A}^{-}+e^{i \theta} s_{B}^{-}\right) \rho_{A B}\right] \\
& =p_{a}+p_{b}+2|\gamma| \cos (\theta+\varphi),
\end{aligned}
$$

where we have extracted and omitted the nonrelevant global phase and kept the relative one, $\theta=k \hat{r} \cdot\left(\vec{R}_{B}-\vec{R}_{A}\right)+\phi_{B}-\phi_{A}$.

Duality under control. Now we are ready to quantify the wave and particle properties of the generated photon. The standard measure of the wave feature is interference visibility related to the superposed atomic state (2) or (3). It can be obtained directly from (5), and is given as

$$
V=\frac{p_{D}^{\max }-p_{D}^{\min }}{p_{D}^{\max }+p_{D}^{\min }}=2|\gamma| .
$$

The particle nature of the photon is embodied by the degree to which it is localized, which in this case means to what degree it is emitted from only one of the two atoms. The 
standard measure is distinguishability, which is represented by the probability difference of the photon being emitted from atoms $A$ and $B$, i.e.,

$$
D=\frac{\left|p_{a}-p_{b}\right|}{p_{a}+p_{b}}=\left|p_{a}-p_{b}\right| .
$$

Due to the fact that $|\gamma| \leqslant \sqrt{p_{a} p_{b}}$, it is straightforward to reach the conventional duality inequality

$$
V^{2}+D^{2}=\left(p_{a}-p_{b}\right)^{2}+4|\gamma|^{2} \leqslant 1 .
$$

As pointed out in Ref. [20], such an inequality is incomplete to represent Bohr's complementarity principle for it embodies neither exclusiveness nor completeness (through $V$ and $D$ ), two characteristic features of complementarity. Here, it is important to note that the controlling parameters $p_{a}, p_{b}, \gamma$ of the duality sum $V^{2}+D^{2}$ correspond only to properties of the atomic source. This indicates that a resolution of the incompleteness of the above inequality (8) needs also to be traced back to the source.

A further analysis of the sum shows that it corresponds solely to the atomic density matrix (3) in the following compact way,

$$
V^{2}+D^{2}=1-4 \operatorname{det} \rho_{A B}=2 \operatorname{Tr} \rho_{A B}^{2}-1 .
$$

One notes that $\operatorname{Tr} \rho_{A B}^{2}$ is the usual state purity measure for (3), varying from $1 / 2$ to 1 , and $2 \operatorname{Tr} \rho_{A B}^{2}-1$ is one form of its normalization. For symmetry considerations and without loss of generality, one can conveniently define a normalized purity as

$$
\mu_{S}=\sqrt{2 \operatorname{Tr} \rho_{A B}^{2}-1} .
$$

This immediately allows us to arrive at the central result of this Rapid Communication, i.e., the Pythagorean duality source relation

$$
V^{2}+D^{2}=\mu_{S}^{2} .
$$

It shows that the wave-particle duality of a photon is controlled by its atomic source, i.e., the complementary behavior of $V$ and $D$ is determined by the source purity $\mu_{S}$. When the source state is maximally mixed $\left(\mu_{S}=0\right)$, the generated photon can display no duality properties at all, i.e., $V=D=0$. When the source is pure $\left(\mu_{S}=1\right)$, the photon can have full wave-particle duality with complete waveness $(V=1)$ and complete particleness $(D=1)$, both reachable.

It is worth noting that investigations of the value of the duality sum $V^{2}+D^{2}$ have been carried out repeatedly [5-8]. Recently, a similar Pythagorean relation called the polarization coherence theorem (PCT) was obtained by Eberly et al. [13], demonstrating connections of the duality sum to the generic degree of polarization within a single classical optical field. The PCT was then extended to generalized two-state distance measures by De Zela [15]. Another important quadratic connection among visibility, coherence, and phase statistics was reported by Luis and co-workers [18]. An equality resembling relation (9) appears in Ref. [17]. However, all these previous results have demonstrated relations of the duality sum with the properties of the quantum object itself. Here, on the other hand, our normalized purity $\mu_{S}$ refers solely to the parameters of the atomic source state, revealing the effect of the source

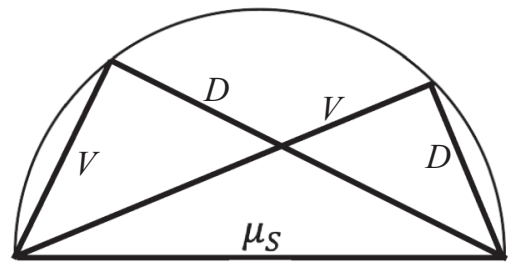

FIG. 2. Geometric illustration of the Pythagorean duality source relation.

rather than the quantum object's other coherence properties on its wave-particle duality behavior.

The quadratic form of measures in (11) indicates its direct connection to the Pythagorean relation. Therefore it can be represented geometrically with right triangles, where the value of $\mu_{S}$ is represented by the length of its longest side and the values of $V, D$ are represented by the two shorter sides. Figure 2 illustrates schematically two examples of such right triangles, who share their longest side (which forms the diameter of a circle) and two corresponding apexes. Both $V$ and $D$ can vary between 0 and $\mu_{S}$, which exhausts all points on the half circle as the right-angle apex.

Generalized result. We now extend the analysis to general and practical situations when additional degrees of freedom of the photon are needed. We first include considerations of vector fields where the polarization degree of freedom matters. When two atoms are not perfectly identical, e.g., the transition between different nearby energy levels, they may emit photons with different polarizations but still with approximately the same energy and spontaneous emission decay rate. Then the description of a photon arriving at the detector needs to be modified as

$$
\begin{aligned}
p_{D} & \propto \operatorname{Tr}\left[\left(\hat{\epsilon}_{a}^{*} s_{a}^{+}+e^{-i \theta} \hat{\epsilon}_{b}^{*} s_{b}^{+}\right)\left(\hat{\epsilon}_{a} s_{a}^{-}+e^{i \theta} \hat{\epsilon}_{b} s_{b}^{-}\right) \rho_{A B}\right] \\
& =p_{a}+p_{b}+2|\gamma \eta| \cos (\theta+\varphi+\Delta),
\end{aligned}
$$

where $\hat{\epsilon}_{a}, \hat{\epsilon}_{b}$ are two respective polarization states of the spontaneously emitted photon from atoms $A, B$, and they have a generic overlap relation $\hat{\epsilon}_{a}^{*} \cdot \hat{\epsilon}_{b}=\eta=|\eta| e^{i \Delta}$. Such a characterization is also consistent with traditional optical polarization analyses $[33,34]$, where a pair of polarizers are used to introduce different polarizations of light coming from two atoms, respectively.

When polarization states are involved, polarizationmodulated total visibility $V_{P}$, proposed by Friberg and coworkers [16,35], is usually employed to represent the full coherence of an optical field. It is defined as

$$
V_{P}=\sqrt{\frac{1}{2} \sum_{j=0}^{3} V_{j}^{2}}, \quad \text { with } \quad V_{j}=\frac{S_{j}^{\max }-S_{j}^{\min }}{S_{0}^{\max }+S_{0}^{\min }} .
$$

Here, $V_{j}, j=0,1,2,3$, are called polarization visibilities [35], and $S_{j}$ are four corresponding conventional polarization Stokes parameters that can be obtained as $S_{j}=\left\langle\hat{S}_{j}\right\rangle$, with $\hat{S}_{j}$ being the Stokes operators that are analogous to Pauli matrices [36]. Recently, this modulated total polarization visibility was employed as a measure of wave property in the complementarity analysis of a quantized vector field (vector single photon) [16]. 
Given the contribution of polarization states, we also adopt this total visibility $V_{P}$ to measure the waveness of the photon. For the physical context here, each individual polarization visibility can be obtained as

$$
V_{0}=V_{1}=2|\gamma \eta|, \quad V_{2}=V_{3}=2|\gamma| \sqrt{1-|\eta|^{2}},
$$

which leads to the total polarization-modulated visibility

$$
V_{P}=2|\gamma|
$$

The photon distinguishability $D$ and atomic state purity $\mu_{S}$ remain the same as in (7) and (10). Then, for the more general and practical case of unbalanced polarizations, one obtains the same form of the above duality Pythagorean relation, i.e.,

$$
V_{P}^{2}+D^{2}=\mu_{S}^{2}
$$

Since $\mu_{S}^{2} \leqslant 1$, this relation explains from another perspective the key relation $V_{P}^{2}+D^{2} \leqslant 1$ obtained in Ref. [16].

In more general and practical cases, the nonperfectness of the atomic source or the influence of external fields and parties may lead to an unbalance of photon states in degrees of freedom other than polarization. Then one can always group the affected one or more degrees of freedom together and represent it with a single (discrete or continuous) state $\left|\phi_{a}\right\rangle$, indicating the photon emitted by atom $A$, and $\left|\phi_{b}\right\rangle$ for atom $B$. Then the probability of photon detection can be described in general as in (12) by replacing $\hat{\epsilon}_{a}, \hat{\epsilon}_{b}$ with $\left|\phi_{a}\right\rangle,\left|\phi_{b}\right\rangle$, respectively. It is important to note that $\left|\phi_{a}\right\rangle,\left|\phi_{b}\right\rangle$ live in an effective two-dimensional space just as do the polarization states $\hat{\epsilon}_{a}, \hat{\epsilon}_{b}$. It is spanned by the basis $\left\{\left|\phi_{a}\right\rangle,\left|\bar{\phi}_{a}\right\rangle\right\}$, where $\left\langle\phi_{a} \mid \bar{\phi}_{a}\right\rangle=0$ and $\left|\phi_{b}\right\rangle$ can always be expressed as $\left|\phi_{b}\right\rangle=$ $\eta\left|\phi_{a}\right\rangle+\sqrt{1-|\eta|^{2}}\left|\bar{\phi}_{a}\right\rangle$. Therefore, one can define a general total modulated visibility $V_{T}$ as in (13) by replacing the polarization Stokes parameters with generic two-dimensional Stokes-like parameters (see a systematic analysis of such parameters by James et al. in Ref. [36]). This further extends the polarization-modulated result (16) to more generalized situations.
Discussion. In summary, we have investigated quantitatively a photon's wave-particle duality in connection with its atomic source. The two-atom point source allows an exact spherical wave analysis that avoids the finite-width issue of conventional double-slit treatments. The amount of information available to be exchanged between waveness and particleness is tunable and determined by the atomic source that gives birth to the quantum object. A general Pythagorean duality source relation is obtained, showing exact quantitative restrictions. Our result opens another way of investigating and understanding duality through the perspective of a quantum object's source.

The consideration of a generic mixed state, through purity $\mu_{S}$, of the atomic source is a practical treatment compatible with experimental conditions. As pointed out by Slodička et al. [28], for their experimental setup, approximately $38 \%$ of the incoherence (or mixedness) of the state (1) comes from imperfect populations, collective magnetic field fluctuations, atomic motion, atomic recoil, etc. Therefore, by tuning some of the properties through cooling or other measures [37], one is able to directly observe the Pythagorean duality source relation (11).

It is worth emphasizing that the overall analysis provided in this Rapid Communication is not limited to photons. It applies to a generic single quantum object that is generated by a two-center source. For example, "Young-type" electron interference was observed in charged-particle-impact ionization of diatomic $\mathrm{H}_{2}$ molecules, due to the superposition of ionization amplitudes associated with the two hydrogen atoms [38]. Also, "spontaneously emitting" an atom was achieved with an optically trapped Bose-Einstein condensate (BEC) [39], and two-path interference was observed with atoms from two BECs [40]. Our analysis also has important implications in the multipath interference of a single quantum object [41] in connection with multicenter source properties such as superradiant and subradiant emissions of multiple entangled atoms [42].

Acknowledgments. We acknowledge conversations with J. H. Eberly. X.-F.Q. acknowledges support from NSF PHY1505189 and PHY-1539859. G.S.A. acknowledges support from AFOSR FA9550-18-1-0141.
[1] L. de Broglie, Waves and quanta, Nature (London) 112, 540 (1923); On the theory of quanta, Ann. Phys. 10, 22 (1925).

[2] N. Bohr, The quantum postulate and the recent development of atomic theory, Naturwissenschaften 16, 245 (1928); The quantum postulate and the recent development of atomic theory, Nature (London) 121, 580 (1928).

[3] W. K. Wootters and W. H. Zurek, Complementarity in the double-slit experiment: Quantum nonseparability and a quantitative statement of Bohr's principle, Phys. Rev. D 19, 473 (1979).

[4] R. J. Glauber, Amplifiers, attenuators, and Schrödinger's cat, Ann. N.Y. Acad. Sci. 480, 336 (1986).

[5] D. M. Greenberger and A. Yasin, Simultaneous wave and particle knowledge in a neutron interferometer, Phys. Lett. A 128, 391 (1988).
[6] L. Mandel, Coherence and indistinguishability, Opt. Lett. 16, 1882 (1991).

[7] G. Jaeger, M. A. Horne, and A. Shimony, Complementarity of one-particle and two-particle interference, Phys. Rev. A 48, 1023 (1993).

[8] G. Jaeger, A. Shimony, and L. Vaidman, Two interferometric complementarities, Phys. Rev. A 51, 54 (1995).

[9] B. G. Englert, Fringe Visibility and Which-Way Information: An Inequality, Phys. Rev. Lett. 77, 2154 (1996).

[10] H. Y. Liu, J. H. Huang, J. R. Gao, M. S. Zubairy, and S.-Y. Zhu, Relation between wave-particle duality and quantum uncertainty, Phys. Rev. A 85, 022106 (2012).

[11] M. Lahiri, Wave-particle duality and polarization properties of light in single-photon interference experiments, Phys. Rev. A 83, 045803 (2011). 
[12] F. De Zela, Relationship between the degree of polarization, indistinguishability, and entanglement, Phys. Rev. A 89, 013845 (2014).

[13] J. H. Eberly, X.-F. Qian, and A. N. Vamivakas, Polarization coherence theorem, Optica 4, 1113 (2017).

[14] F. De Zela, Optical approach to concurrence and polarization, Opt. Lett. 43, 2603 (2018).

[15] F. De Zela, Hidden coherences and two-state systems, Optica 5, 243 (2018).

[16] A. Norrman, K. Blomstedt, T. Setälä, and A. T. Friberg, Complementarity and Polarization Modulation in Photon Interference, Phys. Rev. Lett. 119, 040401 (2017).

[17] M. Jakob and J. A. Bergou, Complementarity and entanglement in bipartite qudit systems, Phys. Rev. A 76, 052107 (2007).

[18] A. Luis, Quantum-classical correspondence for visibility, coherence, and relative phase for multidimensional systems, Phys. Rev. A 78, 025802 (2008).

[19] R. Galazo, I. Bartolomé, L. Ares, and A. Luis, Classical and quantum complementarity, impossible distributions and how much quantumness is truly quantum, arXiv:1811.12636.

[20] X.-F. Qian, A. N. Vamivakas, and J. H. Eberly, Entanglement limits duality and vice versa, Optica 5, 942 (2018).

[21] X.-F. Qian, K. Konthasinghe, S. K. Manikandan, D. Spiecker, A. N. Vamivakas, and J. H. Eberly, Turning off quantum duality, Phys. Rev. Research 2, 012016(R) (2020).

[22] W. K. Wootters, Entanglement of Formation of an Arbitrary State of Two Qubits, Phys. Rev. Lett. 80, 2245 (1998).

[23] B. J. Pearson, N. Ferris, R. Strauss, H. Li, and D. P. Jackson, Measurements of slit-width effects in Young's double-slit experiment for a partially-coherent source, OSA Continuum 1, 755 (2018).

[24] U. Eichmann, J. C. Bergquist, J. J. Bollinger, J. M. Gilligan, W. M. Itano, D. J. Wineland, and M. G. Raizen, Young's Interference Experiment with Light Scattered from Two Atoms, Phys. Rev. Lett. 70, 2359 (1993).

[25] H. Huang, G. S. Agarwal, and M. O. Scully, The interference of radiation from two trapped atoms: Thermal noise and visibility, Opt. Commun. 127, 243 (1996).

[26] W. M. Itano, J. C. Bergquist, J. J. Bollinger, D. J. Wineland, U. Eichmann, and M. G. Raizen, Complementarity and Young's interference fringes from two atoms, Phys. Rev. A 57, 4176 (1998).

[27] C. Cabrillo, J. I. Cirac, P. García-Fernández, and P. Zoller, Creation of entangled states of distant atoms by interference, Phys. Rev. A 59, 1025 (1999).
[28] L. Slodička, G. Hétet, N. Röck, P. Schindler, M. Hennrich, and R. Blatt, Atom-Atom Entanglement by Single-Photon Detection, Phys. Rev. Lett. 110, 083603 (2013).

[29] G. Araneda, D. B. Higginbottom, L. Slodička, Y. Colombe, and R. Blatt, Interference of Single Photons Emitted by Entangled Atoms in Free Space, Phys. Rev. Lett. 120, 193603 (2018).

[30] J. I. Cirac and P. Zoller, Quantum Computations with Cold Trapped Ions, Phys. Rev. Lett. 74, 4091 (1995).

[31] G. S. Agarwal, Quantum Optics, Springer Tracts in Modern Physics Vol. 70 (Springer, Berlin, 1974).

[32] R. Wiegner, J. von Zanthier, and G. S. Agarwal, Quantum interference and non-locality of independent photons from disparate sources, J. Phys. B: At. Mol. Opt. Phys. 44, 055501 (2011).

[33] E. Wolf, Introduction to the Theory of Coherence and Polarization of Light (Cambridge University Press, Cambridge, UK, 2007).

[34] M. Lahiri, A. Hochrainer, R. Lapkiewicz, G. B. Lemos, and A. Zeilinger, Partial polarization by quantum distinguishability, Phys. Rev. A 95, 033816 (2017); M. Krenn, A. Hochrainer, M. Lahiri, and A. Zeilinger, Entanglement by Path Identity, Phys. Rev. Lett. 118, 080401 (2017).

[35] T. Setälä, J. Tervo, and A. T. Friberg, Stokes parameters and polarization contrasts in Young's interference experiment, Opt. Lett. 31, 2208 (2006); L.-P. Leppänen, K. Saastamoinen, A. T. Friberg, and T. Setälä, Interferometric interpretation for the degree of polarization of classical optical beams, New J. Phys. 16, 113059 (2014).

[36] D. F. V. James, P. G. Kwiat, W. J. Munro, and A. G. White, Measurement of qubits, Phys. Rev. A 64, 052312 (2001).

[37] L. Slodička, G. Hétet, N. Röck, S. Gerber, P. Schindler, M. Kumph, M. Hennrich, and R. Blatt, Interferometric thermometry of a single sub-Doppler-cooled atom, Phys. Rev. A 85, 043401 (2012).

[38] D. S. Milne-Brownlie, M. Foster, J. Gao, B. Lohmann, and D. H. Madison, Young-Type Interference in $(e, 2 e)$ Ionization of $\mathrm{H}_{2}$, Phys. Rev. Lett. 96, 233201 (2006).

[39] L. Krinner, M. Stewart, A. Pazmiño, J. Kwon, and D. Schneble, Spontaneous emission of matter waves from a tunable open quantum system, Nature (London) 559, 589 (2018).

[40] M. R. Andrews, C. G. Townsend, H.-J. Miesner, D. S. Durfee, D. M. Kurn, and W. Ketterle, Observation of interference between two Bose condensates, Science 275, 637 (1997).

[41] E. Bagan, J. Calsamiglia, J. A. Bergou, and M. Hillery, Duality Games and Operational Duality Relations, Phys. Rev. Lett. 120, 050402 (2018).

[42] R. Wiegner, J. von Zanthier, and G. S. Agarwal, Quantuminterference-initiated superradiant and subradiant emission from entangled atoms, Phys. Rev. A 84, 023805 (2011). 